\title{
Diffusion in different models of active Brownian motion
}

\author{
B. Lindner ${ }^{\mathrm{a}}$ and E.M. Nicola
}

Max-Planck-Institut für Physik komplexer Systeme, Nöthnitzer Straße 38, 01187 Dresden, Germany

\begin{abstract}
Active Brownian particles (ABP) have served as phenomenological models of self-propelled motion in biology. We study the effective diffusion coefficient of two one-dimensional ABP models (simplified depot model and RayleighHelmholtz model) differing in their nonlinear friction functions. Depending on the choice of the friction function the diffusion coefficient does or does not attain a minimum as a function of noise intensity. We furthermore discuss the case of an additional bias breaking the left-right symmetry of the system. We show that this bias induces a drift and that it generally reduces the diffusion coefficient. For a finite range of values of the bias, both models can exhibit a maximum in the diffusion coefficient vs. noise intensity.
\end{abstract}

\section{Introduction}

Self-propelled motion is one of the most fascinating aspects of biological systems. This motion can appear in many different biological contexts either inside cells or on the multi-cellular level. A typical example of intracellular self-propelled motion is provided by the directed transport of molecular motors along filaments [1,2]. Within an organism, this motion can appear, for instance, in the crawling of cells during wound healing [1]. Moreover, self-propelled motion may appear as a collective property of many organisms, as for example in the movement of whole flocks of animals [3].

Simple phenomenological models may help us to understand the dynamics of self-propelled entities, their statistics and possibly how their dynamics and statistics is related to the biological task (for instance, transport of proteins for molecular motors or food search for the motion of animals). One class of models successfully studied during the last 15 years are active Brownian particles (ABP). This class of models not only take into account random influences on the biological object, dissipation of the objects energy, but also uptake of energy (negative dissipation). The latter is often realized by a friction coefficient which depends nonlinearly on the particle's speed and attains negative values for low speed. The Langevin dynamics for such an active particle with unit mass is given by

$$
\dot{x}=v, \quad \dot{v}=-\gamma(v) v+\sqrt{2 D} \xi(t),
$$

where $\xi(t)$ is Gaussian white noise with $\left\langle\xi(t) \xi\left(t^{\prime}\right)\right\rangle=\delta\left(t-t^{\prime}\right)$ and $D$ is the noise intensity.

For ordinary Brownian motion we would have $\gamma(v)=\gamma_{0}=$ const in Eq. (1) and $D=\gamma_{0} k_{B} T$ (i.e. the Einstein relation, in which $k_{B}$ is the Boltzmann constant and $T$ is temperature). In this case Eq. (1) would describe an equilibrium system. For an active Brownian particle $\gamma(v)$ attains negative values at small speed, has a zero at some finite speed and is positive at large speed. This negative friction turns the model into a nonequilibrium system.

\footnotetext{
a e-mail: benji@pks.mpg.de
} 
The dynamics Eq. (1) and its obvious generalizations to two and three spatial dimensions, to the inclusion of force fields, and to different kinds of coupling of such active particles have been studied extensively in the literature [4-10]. So far, mostly two different friction functions were considered. In the simplified depot model, proposed by Schweitzer, Ebeling, and Tilch in [5] (the so-called SET model), the friction attains the form ${ }^{1}$

$$
\gamma_{S E T}(v)=\gamma_{0}\left[1-\frac{\beta}{1+v^{2}}\right] .
$$

This nonlinear friction gives rise to self-propelled motion if $\beta>1$; this friction function has zeros at $v= \pm \sqrt{\beta-1}$, is negative at speeds between and positive beyond these values. Another popular choice for a negative dissipation function is the Rayleigh-Helmholtz friction (RH model in the following) given by

$$
\gamma_{R H}(v)=\gamma_{0}\left[v^{2}-\alpha\right]
$$

with $\alpha>0$. Indeed, for $\alpha>0$ the friction is negative between and positive beyond the zeros at $\pm \sqrt{\alpha}$. The $\mathrm{RH}$ nonlinear friction has its origin in the work of Rayleigh and Helmholtz on the propagation of sound [11,12] and was extensively studied by Klimontovich [13]. In the context of ABP, it has been studied in, e.g. [6,8,14]. A third model (not addressed here) that has attracted some attention was proposed by Schienbein and Gruler in [4]. In this model the friction depends piecewise linearly on the velocity, with a discontinuity at $v=0$.

So far, most studies of active Brownian particles assumed a symmetric function $\gamma(v)$. There are several reasons why an asymmetric function could be of interest. First of all and from a general point of view, the symmetric (isotropic) case is certainly not generic: molecular motors have a preferred direction of motion along filaments, cells are not always rotationally symmetric which may lead in conjunction with their internal force-generating mechanisms to a bias towards a specific direction, etc. Secondly, active Brownian particles describe biological entities that are not isolated in an empty space but influenced by their environment; the simplest effect may be described by a bias force ${ }^{2}$. Thirdly, even if the ABP dynamics is isotropic, it might be interesting how it responds to external influences which break the symmetry of the system; in other words, the linear and nonlinear response to static stimuli may reveal dynamical aspects otherwise not accessible experimentally. In particular, we will consider in this paper extentions of the SET and RH models, where we break the symmetry of the system with a simple constant bias $F$ :

$$
\dot{x}=v, \quad \dot{v}=-\gamma(v) v+F+\sqrt{2 D} \xi(t) .
$$

The central quantity for a Brownian motion - be it active or passive, biased or unbiased - is the (effective) diffusion coefficient defined by

$$
D_{\mathrm{eff}}=\lim _{t \rightarrow \infty} \frac{1}{2 t}\left\langle[x(t)-\langle x(t)\rangle]^{2}\right\rangle
$$

where the brackets stand for the average over an ensemble of trajectories (please note that we have included the case of finite transport by subtracting the time-dependent mean value). We would like to point out that the diffusion coefficient is related to the variance $\left\langle\Delta v^{2}\right\rangle$ and the correlation time of the velocity $\tau_{\text {corr }}$ through the Kubo relation as follows

$$
D_{\mathrm{eff}}=\left\langle\Delta v^{2}\right\rangle \tau_{c o r r}
$$

where $\tau_{\text {corr }}=\int_{0}^{\infty} d \tau\left[\langle v(t) v(t+\tau)\rangle-\langle v(t)\rangle^{2}\right] /\left\langle\Delta v^{2}\right\rangle$. The importance of $D_{\text {eff }}$ is evident if we consider Fig. 1 where an ensemble of trajectories of the RH model is shown as an example with and without bias.

\footnotetext{
1 Note that additional parameters present in the original model can be set to one by a rescaling of $x$ and $v$.

2 The other obvious simple choice would be a harmonic potential as provided by a spring; this has been studied by Ebeling et al. [5,7].
} 

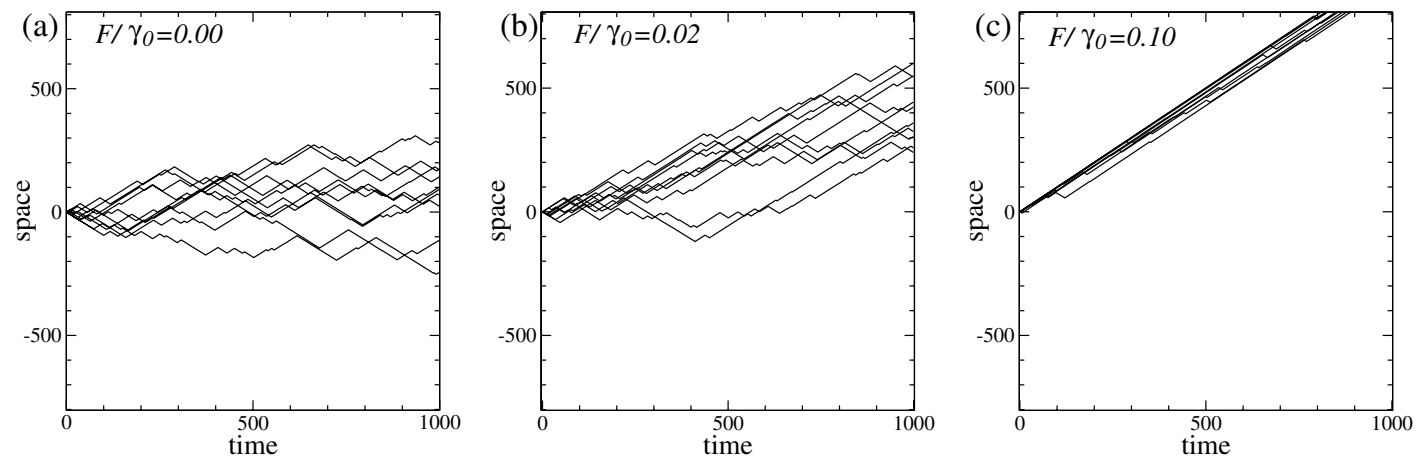

Fig. 1. Examples of the trajectories of the RH model obtained by numerical integration of Eqs. $(3,4)$ with noise intensity $D=1$ and bias strength $F / \gamma_{0}=0.00,0.02$ and $0.10 \mathrm{in}$ (a), (b) and (c) respectively (10 trajectories are shown in each panel, all of them starting from the same location and parameters are $\gamma_{0}=20$ and $\alpha=1$ ). Note that the dynamics is effectively diffusive with a constant drift.

For the unbiased SET model $(F=0)$ it was recently shown [15] that the diffusion coefficient displays a minimum as a function of the noise intensity $D$. We recall that for ordinary Brownian motion the diffusion coefficient is directly proportional to the noise intensity $D_{\text {eff }}=D / \gamma_{0}^{2}$. The minimum in the diffusion coefficient of the active particle implies that we may localize an $A B P$ in space by adding noise - an effect reminiscent of stochastic resonance where fluctuations can also play a constructive role [16]. Such a noise-induced localization may help optimizing food searches or the performance of other tasks.

The first main question addressed in this paper is whether such a minimum of the diffusion coefficient is robust or whether it depends on the choice of the model. This question will be inspected by comparing diffusion coefficients of the SET and RH models.

The second question studied in this paper is how the diffusion in the two ABP models changes when a constant bias $F$ is included. In particular, we are again interested how the diffusion coefficient $D_{\text {eff }}$ looks like as a function of the noise intensity $D$. We note that in case of a finite bias, the system also shows transport (i.e. unlike in the unbiased case, the mean velocity $\langle v\rangle$ is not zero anymore for $F \neq 0$ ).

This paper is organized as follows. In the next section we briefly study the deterministic dynamics of both models including their bifurcations. In section 3 we discuss the unbiased diffusion in the SET and RH models. In section 4, we address what happens to the diffusion if a bias force is added. Our results are summarized in section 5, where an outlook on directions of possible future research is given.

\section{The models and their deterministic dynamics}

In this section we will shortly discuss the dynamics of the SET and RH models (given by Eqs. $(2,4)$ and $(3,4)$ respectively) without noise.

Let us firstly note that Eq. (4) can be rewritten

$$
\dot{v}=-U^{\prime}(v)+\sqrt{2 D} \xi(t),
$$

where the prime denotes the derivative with respect to $v$ and where the effective velocity potential is given by

$$
U(v)=\int^{v} d \tilde{v}[\gamma(\tilde{v}) \tilde{v}-F] .
$$

The dynamics of the system will be such that, after a transient, the velocity $v$ relaxes to one of the minima of this potential. Furthermore, in the next sections we will see that this potential 

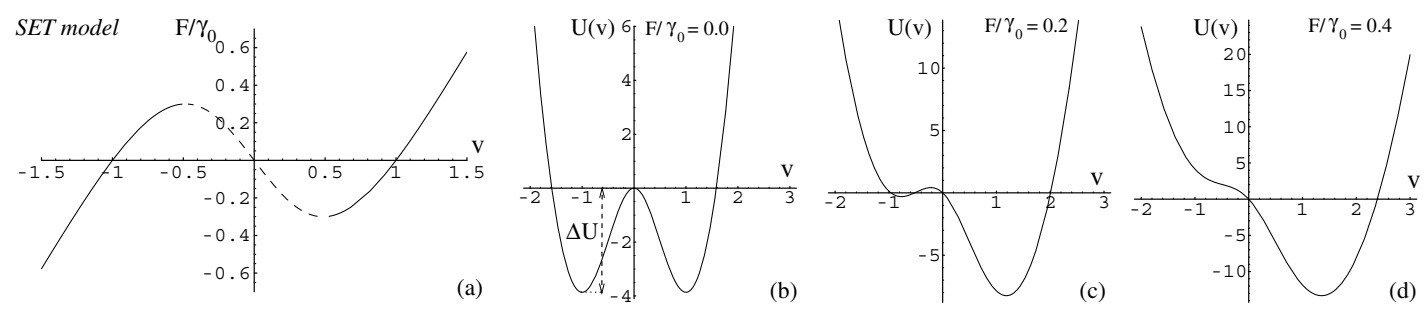

Fig. 2. (a) Fixed points of the velocity of the SET model for $\beta=2$ (and $\gamma_{0}=20$ ). If $\left|F / \gamma_{0}\right|<0.300283$ two stable solutions (left and right solid lines) exist. One of them disappears through a saddle node bifurcation for $F_{S E T}^{\text {bif }}$. (b,c,d) Velocity potentials for the SET model according to Eq. (9) for three characteristic values of the bias $F\left(F / \gamma_{0}=0\right.$ in (b), 0.2 in (c) and 0.4 in (d)). The plot in (a) gives the positions of the minima (solid lines) and the maximum (dashed line) for a certain bias force.
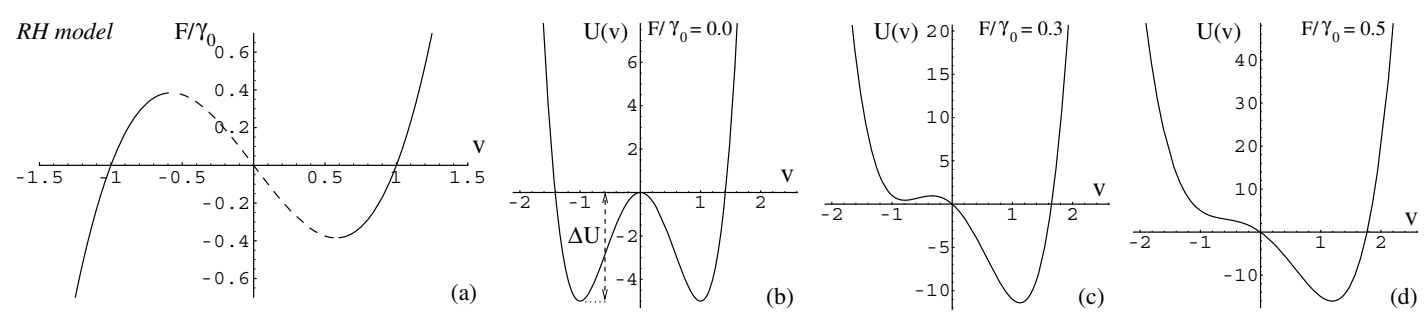

Fig. 3. (a) Fixed points of the velocity of the RH model for $\alpha=1$ (and $\gamma_{0}=20$ ). If $\left|F / \gamma_{0}\right|<$ 0.384900 two stable solutions (left and right solid lines) exist. One of them disappears through a saddle node bifurcation for $F_{R H}^{\text {bif }}$. (b-d) Velocity potentials for the RH model according to Eq. (9) for three characteristic values of the bias $F\left(F / \gamma_{0}=0\right.$ in (b), 0.3 in (c) and 0.5 in (d)). The plot in (a) gives the positions of the minima (solid lines) and the maximum (dashed line) for a certain bias force.

is also helpful to understand the dynamics of the system under noise (in particular, for weak noise, see below).

The explicit potentials for the SET and the RH model read

$$
U_{S E T}(v)=\gamma_{0}\left(\frac{v^{2}}{2}-\frac{\beta \ln \left[1+v^{2}\right]}{2}\right)-F v
$$

(cf. [10]) and

$$
U_{R H}(v)=\gamma_{0}\left(\frac{v^{4}}{4}-\alpha \frac{v^{2}}{2}\right)-F v
$$

respectively. Several examples of possible potential shapes are shown in Figs. 2(b-d) and 3(b-d). In Figs. 2(a) and 3(a) the location of the minima (full-line) and of the maximum (dashed-line) are shown as the bias $F$ is changed (note that both models are odd with respect to $F$; consequently only positive values of $F$ need to be considered). For small values of this bias, a bistable velocity potential exists. Consequently, two possible values of the velocity can occur. This happens if $-F^{\text {bif }}<F<F^{\text {bif }}$, where

$$
F_{S E T}^{\mathrm{bif}}=\frac{\gamma_{0}}{2} \frac{(3 \beta-\lambda) \sqrt{2(\lambda-\beta-2)}}{\lambda-\beta}, \quad \lambda=\sqrt{\beta(\beta+8)},
$$

for the SET model and

$$
F_{R H}^{\text {bif }}=2 \gamma_{0}\left(\frac{\alpha}{3}\right)^{3 / 2}
$$


for the RH model. With $\beta=2$ and $\alpha=1$ we obtain $F_{S E T}^{\text {bif }} / \gamma_{0}=0.300283$ and $F_{R H}^{\text {bif }} / \gamma_{0}=$ 0.384900 for the SET and RH models, respectively. Each of these bifurcation is of the saddlenode type ${ }^{3}$.

In the unbiased case $(F=0)$, the potential is symmetric about the origin and bistable in both cases. Note the differences in the potential barrier at $v=0$ in Figs. 2(b) and 3(b), and in the curvature at minima and maxima. We also would like to point out that the potentials differ in their asymptotic behavior. For large $|v|$, the square term dominates in the SET model, corresponding to normal Stokes friction, i.e. a friction force proportional to the velocity $v$. The potential of the RH model shows a much stronger increase with $v^{4}$, corresponding to a friction force proportional to $v^{3}$.

Our standard parameter values used throughout the following are

$$
\gamma_{0}=20, \quad \alpha=1, \quad \beta=2,
$$

which ensure that the two friction functions have both their zeros at \pm 1 for vanishing bias $(F=0)$.

\section{Diffusion of unbiased active Brownian particles}

If the bias force is switched off $(F=0)$, the problem simplifies considerably. In fact for this case, i.e. the free nonlinear Brownian motion with an even friction function, the diffusion coefficient has been recently analytically calculated [15] (including also the case that the noise intensity is an even function of velocity). The result can be written in terms of quadratures of the velocity potential $U(v)$ of Eqs. (9) and (10) with $F=0$.

The quadrature result from Ref. [15] reads for our problem (where only additive noise is involved)

$$
D_{\text {eff }}=\frac{\int_{0}^{\infty} d v_{2} e^{U\left(v_{2}\right) / D}\left[\int_{v_{2}}^{\infty} d v_{1} e^{-U\left(v_{1}\right) / D} v_{1}\right]^{2}}{D \int_{0}^{\infty} d v_{3} e^{-U\left(v_{3}\right) / D}} .
$$

This result can be evaluated numerically for the two models. In Fig. 4 we compare curves obtained in this way (solid line) with numerical simulations of the full model (symbols). There is a qualitative difference between the dependence of the diffusion coefficient on noise intensity in the two models. As already shown in [15], the SET model ( $c f$. Fig. 4(a)) displays a minimum versus noise intensity. Both for $D$ approaching zero and infinity the diffusion coefficient diverges. The model with RH model ( $c f$. Fig. 4(b)) does not show a minimum - here the diffusion coefficient decreases monotonically with growing noise intensity saturating in the strong noise limit at a finite value. In order to understand why a minimum occurs in the SET but not in the RH model we now discuss the asymptotic behavior at weak and strong noise.

For weak noise the behavior of both models is similar. The velocity is essentially close to one of the two minima of the bistable velocity potential ( $c f$. Figs. 2(b) and 3(b)). Because these minima lay at finite speed, the active Brownian particle goes straight to the right (left) as long as the velocity resides in the right (left) minimum. Transitions between velocity states are rare with an exponential waiting time density. Hence, the velocity performs the classical telegraph noise [17] and the corresponding spatial diffusion coefficient is inversely proportional to the hopping rate between the two potential minima $[8,18,19]$

$$
D_{\text {eff }}=\frac{v_{0}^{2}}{2 r_{k}}
$$

\footnotetext{
${ }^{3}$ Note that if we additionally consider the parameter measuring the distance to the bifurcation to a self-propelled motion (i.e., $\beta$ for SET model and $\alpha$ for RH model), a cusp codimension- 2 bifurcation is observed.
} 

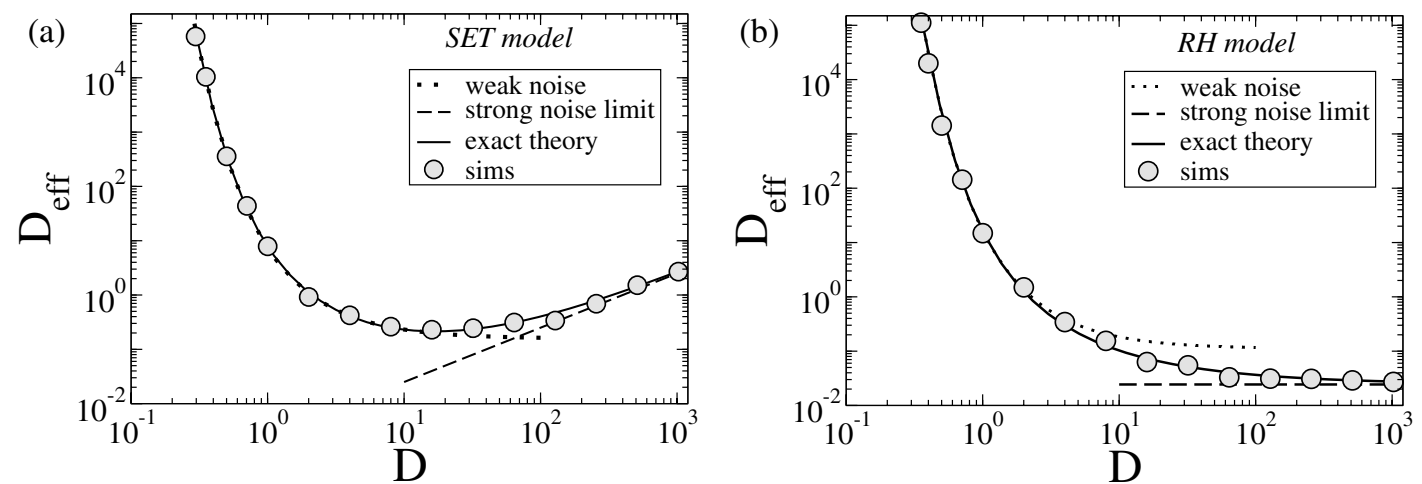

Fig. 4. Diffusion coefficient $D_{\text {eff }}$ as a function of noise intensity $D$ for the SET model (a) and the RH model (b). Simulations (circles) were done with different time steps (smaller at strong noise) ranging from $10^{-4}-10^{-2}$ a.u. for $t_{\text {sim }}=10^{6}$ and the diffusion coefficient was estimated from 100 realizations of the process.

where $v_{0}$ is the speed at the symmetric potential minima and $r_{k}$ is the Kramers rate for the overdamped case

$$
r_{k}=\frac{\sqrt{U^{\prime \prime}\left(v_{0}\right)\left|U^{\prime \prime}(0)\right|}}{2 \pi} \exp [-\Delta U / D] .
$$

The weak noise result Eq. (15) is shown in Fig. 4(a) and (b) by dotted lines. It fits well to both simulations and the exact analytical result for both the SET and RH models. We note that the weak noise result can be also obtained from a saddle-point approximation of the exact result.

Why does the diffusion coefficient diverge in the limit of vanishing noise? In this limit the hopping rate between the metastable states goes to zero. Thinking in terms of an ensemble average: half of the ensemble would be at $v=v_{0}$, going straight to the right. The other half is at $v=-v_{0}$ and thus goes straight to the left. The mean distance in this case would grow linearly in time, the squared distance or mean square displacement like $t^{2}$. Such ballistic motion then manifests itself in a diverging diffusion coefficient because the diffusion coefficient measures the linear growth of the mean square displacement. The divergence of the diffusion coefficient in the zero-noise limit is thus a consequence of the finite speed (ballistic motion) in the deterministic case and can thus also be expected in higher spatial dimensions.

For strong noise, the behavior of the models differ - the diffusion coefficient either grows linearly with noise strength (SET model) or saturates (RH model). This can be understood by the different asymptotics of the velocity potentials because for strong fluctuations the velocity is expected to attain large speed values most of the time. Put differently, the existence of the potential barrier at $v=0$ becomes immaterial to the diffusion problem, the particle "does not feel" the barrier anymore if fluctuations become very strong $(D \gg \Delta U)$. For the SET model the parabolic part of the potential dominates at large $v$; this parabolic potential corresponds to normal Stokes friction and we thus expect that for strong noise the diffusion coefficient behaves like for normal ("passive") Brownian motion

$$
D_{\text {eff }}=D / \gamma_{0}^{2}, \quad D \gg \Delta V .
$$

Indeed, the exact result as well as the simulation result converge to this linear function (dashed line in Fig. 4(a).

For the RH friction, however, the asymptotics is different because at large speed a $v^{4}$ term dominates. We can calculate the diffusion coefficient for a pure $v^{4}$ potential (dropping the $v^{2}$ term and thus the bimodal character of the potential at small speed) from Eq. (14). The result

$$
D_{\text {eff }}=\gamma_{0}^{-1}\left(\frac{1}{4}\right)^{1 / 4} \Gamma\left(\frac{3}{4}\right) \int_{0}^{\infty} d x \operatorname{erfc}^{2}\left(x^{2}\right) e^{x^{4}} \approx 0.4875 \gamma_{0}^{-1},
$$



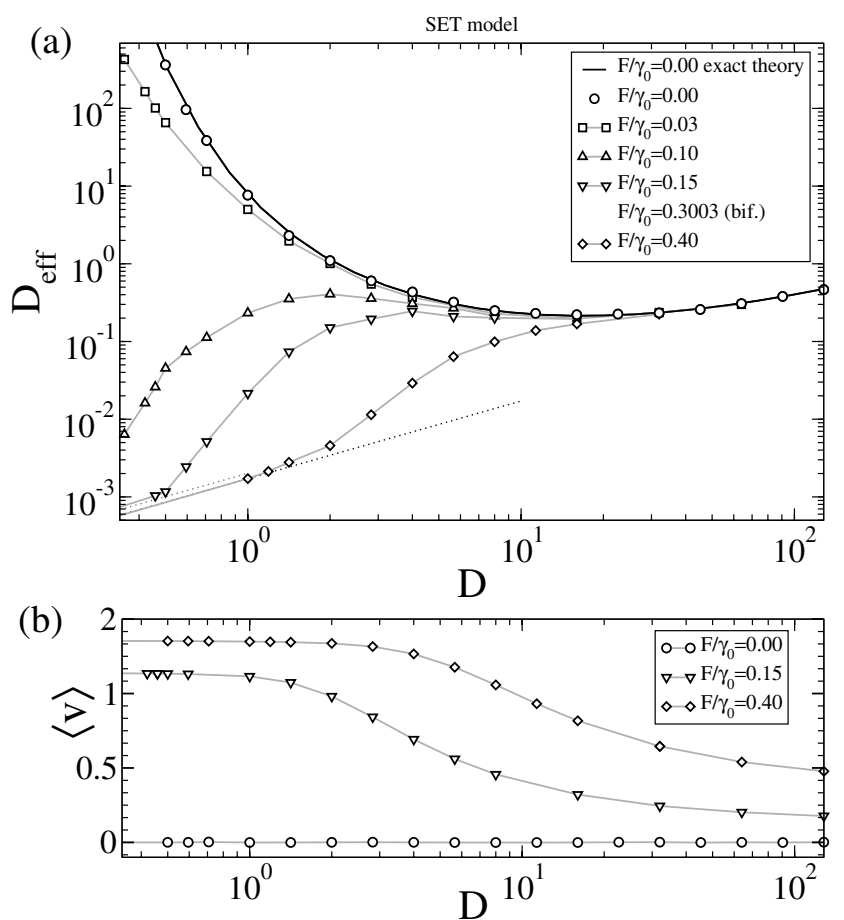

Fig. 5. (a) Effective diffusion coefficient $D_{\text {eff }}$ for the SET model with a constant bias term of strength $F$ (recall that the deterministic bifurcation occurs at $F / \gamma_{0}=0.300283$ ). Regardless of the force strength $F$, all curves merge in the strong noise limit. In this limit, $D_{\text {eff }}$ grows linearly with $D$. For $F>F_{S E T}^{\text {bif }}$ the value of $D_{\text {eff }}$ increases monotonically with growing $D$ (see discussion in the main text). For bias values in the range $0<F<F_{S E T}^{b i f}$, the effective diffusion $D_{\text {eff }}$ for weak noise intensities $D$ depends strongly on the value of the bias $F$. The dotted lines indicate the asymptotic diffusion approximation $D /\left|U^{\prime \prime}\left(v_{\min , R}\right)\right|^{2}$ discussed in the main text. (b) Mean velocity $\langle v\rangle$ for the unbiased and biased SET model. In the biased case $\langle v\rangle$ decreases monotonically with the noise intensity $D$. To calculate $D_{\text {eff }}$ we have numerically integrated the system with a time step $\Delta t$ being either 0.001 or 0.01 (for large and small $D$, respectively), with a total integration time of $10^{5}$. To estimate the value of $D_{\text {eff }}$ we have averaged over 1000 realizations. The ensemble of initial velocities chosen for the realizations is critical in the weak noise limit with bias. In our simulations the initial velocity of the first realization is chosen to be positive. For the subsequent realizations the last velocity of the previous run is used as the initial velocity. The total time is always chosen long enough such that hundreds of transitions between both velocity minima take place during the numerical simulation.

is surprising because for such a system the diffusion coefficient does not depend on the noise at all [20]. In our present context it means that the diffusion coefficient will saturate at a finite (small) value as the noise strength approaches infinity. In general we cannot conclude (but also not exclude) the appearance of a minimum from the asymptotic limits. Of course, we know from the exact solution that there is no minimum.

Summarizing, the SET model shows already in the case of free diffusion a remarkable stochastic-resonance like behavior: the diffusion coefficient undergoes a minimum as a function of noise intensity. The RH model in turn does not show the same effect. This is due to the different behavior of both models for high speed. If the high-speed limit implies Stokes friction (as for the SET model), a minimum can be expected. The latter will not be present for a friction force that has a nonlinear high-speed asymptotics $F_{\text {friction }} \sim|v|^{\alpha}$ with $\alpha \geq 3$.

\section{Effective diffusion with a constant bias}

In order to address the effect of the bias $F$ we have performed extensive numerical simulations of both models and measured $D_{\text {eff }}$ and $\langle v\rangle$. 

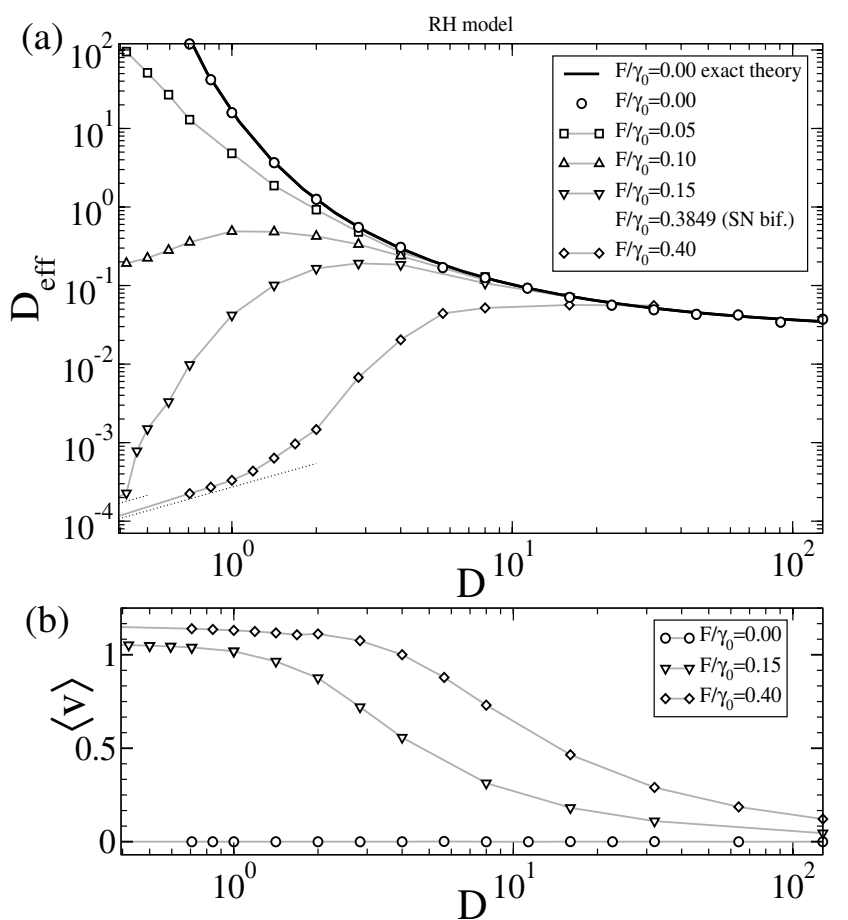

Fig. 6. (a) Effective diffusion coefficient $D_{\text {eff }}$ for the $\mathrm{RH}$ model with a constant bias $F$. The deterministic bifurcation occurs at $F / \gamma_{0}=0.384900$. For very small bias the diffusion coefficient still diverges ( $c f$. data for $F / \gamma_{0}=0.05$ ). For larger bias the diffusion coefficient grwos from zero and attains a maximum at finite noise intensity. In this case at low noise the simple estimate for $D_{\text {eff }}$ given in the text (dotted lines) agrees well with the simulation results. Note also that the bias becomes irrelevant for strong $D$ where $D_{\text {eff }}$ saturates at a constant value. (b) Mean velocity $\langle v\rangle$ for the unbiased and biased RH model. As for the SET model, we obtain a monotonic decrease with growing noise. The simulation method and parameters are the same as for the SET model, see caption of Fig. 5.

In general, a finite bias $F$ induces a transport $\langle v\rangle \neq 0$ towards the direction of the bias (see Figs. 5(b) and 6(b)). The mean velocity $\langle v\rangle$ decreases monotonically with the noise intensity $D$ as long as the bias is positive. It starts for weak noise at the velocity which corresponds to the right minimum of the velocity potential $U(v)$ (for a subcritical bias $F<F^{b i f}$ ) or simply to the only minimum of the velocity potential (for a supracritical bias $F>F^{b i f}$ ). For weak noise this minimum is the state (in velocity space) that the particle occupies almost all the time. For increasing noise the particle spends more and more of its time also at negative velocities - this clearly diminishes the mean velocity.

Our simulations indicate that a finite bias always reduces the effective diffusion coefficient. The resulting dependence of $D_{\text {eff }}$ on the noise intensity $D$ for different values of the bias $F$ is presented in Figs. 5(a) and 6(a) for the SET and RH models, respectively. We can distinguish two well defined limits: strong and weak noise.

In the strong noise limit the effect of the bias is irrelevant since the velocity attains larger and larger (positive and negative) values at which the nonlinear friction $v \gamma(v)$ is always much stronger than the bias $F$. The effective diffusion $D_{\text {eff }}$ just follows the behavior already found in the previous section for $F=0$; a linear growth for the SET model and a saturation to a constant value for the $\mathrm{RH}$ model. Consequently, all curves merge at large noise intensity.

At weak noise the diffusive behavior depends strongly on the exact value of the bias. For sufficiently small bias we obtain curves that still seem to diverge in the zero-noise limit both for the SET model ( $c f$. the data for $F / \gamma_{0}=0.03$ ) and the RH model ( $c f$. the data for $F / \gamma_{0}=0.05$ ). For the SET model these data still pass through a minimum at a high noise intensity.

For higher values of the bias (but still in the regime where two potential minima exist) the diffusion coefficient drops to zero in the limit of vanishing noise for both models. Depending on 
the exact value of this large bias the diffusion coefficient of the SET model can either show a maximum (attained at lower $D$ ) and a minimum (attained at higher $D$ ) or a monotonic increase ( $c f$. data for $F / \gamma_{0}=0.4$ ). The RH model at sufficiently high bias (such that we do not observe a divergence in the zero-noise limit) seems to always show a maximum of $D_{\text {eff }}$ vs. noise intensity. For sufficiently strong bias (when the diffusion coefficient does not diverge), we may also give an estimate using the approximation of a velocity performing an Ornstein-Uhlenbeck process around the right (or the only) potential minimum with curvature $U^{\prime \prime}\left(v_{\min , R}\right)$. Such a process would have the diffusion coefficient $D_{\text {eff }}=D /\left|U^{\prime \prime}\left(v_{\min , R}\right)\right|^{2}$ which describes the simulation data at weak noise reasonably well ( $c f$. the dotted lines in Fig. 5(a) and Fig. 6(a)).

\section{Conclusions}

We have shown that there are qualitative differences for the diffusion in different models of active Brownian particles. While the SET model shows a minimal diffusion at finite noise intensity, an active Brownian particle with $\mathrm{RH}$ friction shows minimal diffusion in the limit of infinite noise intensity. We could trace back this difference to the asymptotics of the friction function at high speed.

The behavior of both models becomes more complicated if the symmetry of the system is broken by a bias force $F$. First of all, with a finite bias both models show a finite transport with a nonzero mean velocity and a general reduction of the effective diffusion. More remarkable, for an intermediate bias both models can show a maximum of the diffusion coefficient vs. noise intensity. In the SET model we will still see a minimum appearing at higher noise intensity.

We also showed that the features added by a symmetry-breaking bias are qualitatively the same for both ABP models since they appear for weak-to-moderate noise where the different asymptotics of the friction functions is immaterial to the diffusion problem.

In biological systems it may be very important to minimize or maximize diffusion. In this paper we have shown that the effective diffusion can be tuned by either the noise intensity or a bias. Given that the parameters of a biological object are subject to evolution one may speculate that for certain tasks requiring minimal effective diffusion the internal noise has been optimized to those discussed here in this paper.

Also of central importance in biology is the question of how to distinguish which ABP model is more appropriate for the description of a particular experiment. Adding noise to a self-propelled object is certainly not simple, however, if it would be possible adding mechanical fluctuation to a crawling cell or moving bacteria, the dependence of the diffusion coefficient on this added noise may give hints to an appropriate effective friction function. If it is possible to add noise an additional static bias $F$ will not be a problem either and yields another opportunity to estimate the correct friction function as well as the level of internal "biological" noise.

Motivated by the problem of molecular motors, we focused in this paper exclusively on onedimensional active Brownian particles. How much of our results does apply to multi-dimensional cases interesting in other context? For the strong-noise case we can refer to Ref. [20] where it was shown that the power law dependence of the diffusion coefficient on noise intensity remains the same as in one-dimensional systems. At weak noise, the diffusion of active particles is dominated by phase diffusion [6] which still (at least in the case of a vanishing bias) leads to a diverging diffusion coefficient. In a $2 \mathrm{~d}$ system in the absence of a bias we have, for instance, $D_{\text {eff }} \sim D^{-1}$ for weak noise [6]; so for the SET model we still expect a minimum in the diffusion coefficient vs. noise intensity because the diffusion coefficient goes to infinity for both $D \rightarrow 0$ and $D \rightarrow \infty$. How the diffusive behavior at weak noise changes if particles in $2 \mathrm{~d}$ are biased towards a certain direction is not evident but remains an interesting open question.

Another interesting problem is to consider detailed microscopic models which map to the dynamics Eq. (4) and try to estimate the noise parameter in terms of the parameters of the microscopic model. These questions will be addressed in a forthcoming publication.

We would like to thank the referee of this manuscript for valuable hints regarding the importance of the initial conditions in the case of biased active Brownian motion. 


\section{References}

1. D. Bray, Cell Movements (Garland, New York, NY, 2001)

2. J. Howard, Mechanics of Motor Proteins and the Cytoskeleton (Sinauer Associates, Sunderland, Mass., 2001)

3. J.K. Parrish, W.M. Hamner, Animal Groups in Three Dimensions (Cambridge University Press, Cambridge, UK, 1997)

4. M. Schienbein, H. Gruler, Bull. Math. Biol. 55, 585 (1993)

5. F. Schweitzer, W. Ebeling, B. Tilch, Phys. Rev. Lett. 80, 5044 (1998)

6. A.S. Mikhailov, D. Meinköhn, Stochastic Dynamics, edited by L. Schimansky-Geier T. Pöschel (Springer, Berlin, London, New York, 1998), p. 334

7. U. Erdmann, W. Ebeling, L. Schimansky-Geier, F. Schweitzer, Eur. Phys. J. B 15, 105 (2000)

8. M. Badoual, F. Jülicher, J. Prost, PNAS 99, 6696 (2002)

9. U. Erdmann, W. Ebeling, L. Schimansky-Geier, A. Ordemann, F. Moss (2004) http://arxiv.org/abs/q-bio.PE/0404018

10. W. Ebeling, Cond. Matter Phys. 7, 539 (2004)

11. J.W.S. Rayleigh, The Theory of Sound (Mac-Millan, London, 1894)

12. H. Helmholtz, On the Sensations of Tone (Dover Publications, New York, 1954)

13. Yu.L. Klimontovich, Statistical Theory of Open Systems (Kluwer Academic Publishers, Dordrecht, Boston, London, 1995)

14. L. Schimansky-Geier, U. Erdmann, N. Komin, Phys. A 351, 51 (2005)

15. B. Lindner, New J. Phys. 9, 136 (2007)

16. L. Gammaitoni, P. Hänggi, P. Jung, F. Marchesoni, Rev. Mod. Phys. 70, 223 (1998)

17. C.W. Gardiner, Handbook of Stochastic Methods (Springer-Verlag, Berlin, 1985)

18. J.M. Sancho, J. Math. Phys. 25, 354 (1984)

19. B. Lindner, Physik Irreversibler Prozesse und Selbstorganisation, edited by T. Pöschel, H. Malchow, L. Schimansky-Geier (Logos-Verlag, Berlin, 2006)

20. B. Lindner, J. Stat. Phys. (2007) (in press) 